\title{
Assessment of Complex System Dynamics via Harmonic Mapping in a Multifractal Paradigm
}

\author{
Gabriel Gavriluț ${ }^{1}$, Liliana Topliceanu ${ }^{2}$, Manuela Gîrțu ${ }^{3}$, Ana Maria Rotundu ${ }^{1}$ (D) Stefan Andrei Irimiciuc ${ }^{4,5}, *$ (D) \\ and Maricel Agop 5,6 \\ 1 Faculty of Phisics, Alexandru Ioan Cuza University, Bulevardul Carol I nr. 11, 700506 Iași, Romania; \\ gavrilutgabriel@yahoo.com (G.G.); anabotezatu82@gmail.com (A.M.R.) \\ 2 Faculty of Engineering, Vasile Alecsandri University of Bacau, 600115 Bacau, Romania; lili@ub.ro \\ 3 Department of Mathematics and Informatics, Vasile Alecsandri University of Bacau, 600115 Bacau, Romania; \\ girtum@yahoo.com \\ 4 National Institute for Laser, Plasma and Radiation Physics, 409 Atomistilor Street, 077125 Bucharest, Romania \\ 5 Romanian Scientists Academy, 54 Splaiul Independentei, 050094 Bucharest, Romania; magop@tuiasi.ro \\ 6 Department of Physics, "Gh. Asachi" Technical University of Iasi, 700050 Iasi, Romania \\ * Correspondence: stefan.irimiciuc@inflpr.ro
}

check for updates

Citation: Gavrilut, G.; Topliceanu, L.; Gîrțu, M.; Rotundu, A.M.; Irimiciuc, S.A.; Agop, M. Assessment of Complex System Dynamics via Harmonic Mapping in a Multifractal Paradigm. Mathematics 2021, 9, 3298. https://doi.org/10.3390/math 9243298

Academic Editors: Catalin I. Pruncu and Maria Luminița Scutaru

Received: 30 November 2021 Accepted: 17 December 2021 Published: 18 December 2021

Publisher's Note: MDPI stays neutral with regard to jurisdictional claims in published maps and institutional affiliations.

Copyright: (c) 2021 by the authors. Licensee MDPI, Basel, Switzerland. This article is an open access article distributed under the terms and conditions of the Creative Commons Attribution (CC BY) license (https:/ / creativecommons.org/licenses/by/ $4.0 /)$.

\begin{abstract}
In the present paper, nonlinear behaviors of complex system dynamics from a multifractal perspective of motion are analyzed. In the framework of scale relativity theory, by analyzing the dynamics of complex system entities based on continuous but non-differentiable curves (multifractal curves), both the Schrödinger and Madelung scenarios on the holographic implementations of dynamics are functional and complementary. In the Madelung scenario, the holographic implementation of dynamics (i.e., free of any external or internal constraints) has some important consequences explicated by means of various operational procedures. The selected procedures involve synchronous modes through SL (2R) transformation group based on a hidden symmetry, coherence domains through Riemann manifold embedded with a Poincaré metric based on a parallel transport of direction (in a Levi Civita sense). Other procedures used here relate to the stationary-non-stationary dynamics transition through harmonic mapping from the usual space to the hyperbolic one manifested as cellular and channel type self-structuring. Finally, the Madelung scenario on the holographic implementations of dynamics are discussed with respect to laser-produced plasma dynamics.
\end{abstract}

Keywords: harmonic mapping; complex system dynamics; SL (2R) group; hidden symmetries

\section{Introduction}

Nonlinearity is accepted as one of the most fundamental properties of any complex system dynamics. Interactions between the structural units of any complex system imply mutual constraints at different scale resolution. Then, the universality of the dynamics laws for any complex system becomes natural and must be reflected in various theoretical models that could describe their dynamics. Some of the usual theoretical models are based on the hypothesis that the variables characterizing the complex system dynamics are differentiable, which can be otherwise unjustified. In such a perspective, the validations of the previously described type of models need to be seen as sequential and applicable on restricted domains for which integrability and differentiability are respected. Since nonlinearity implies predominantly non-differentiable behaviors in the description of complex system dynamics, it is necessary to explicitly introduce the scale resolution in the equations defining the dynamics-governing variables. It implies that any variables used in the description of any complex system now have a dual dependence on the space-time coordinates and the scale resolution. In this new perspective, for instance, instead of using variables defined by non-differentiable functions, approximations of these complex functions that will be used at various scale resolutions are becoming available and 
operational. Therefore, all variables used to define the complex system dynamics will work as a limit of families of functions, which for a null scale resolution are non-differentiable but for non-null scale resolution are differentiable. The previous mathematical procedure involves the development of suitable geometrical structures and a class of models for which the motion laws are integrated with the scale laws. Such geometrical structures are built on the concept of multifractality, and the equivalent theoretical models are based on the scale relativity theory, either with the fractal dimension $D_{f}=2$ (standard model) or in an arbitrary and constant dimension (multifractal theory of motion). In this class of models (non-differentiable), the complex system's structural unit's dynamics can be described by continuous but non-differentiable movement curves (multifractal motion curves). These curves exhibit self-similarity as their main property at any of the points forming the curve, which translates into behaviors of holographic type (every part reflects the global system). Such a complex approach suggests that only holographic implementations can offer complete descriptions of the complex system dynamics [1-3].

According to our previous report from [4], by assimilating any complex fluid with a mathematic object of fractal type in the framework of scale relativity theory (SRT) [5], various non-linear behaviors through a fractal hydrodynamic-type description as well as through a fractal Schrodinger-type description, were established. Thus, the fractal hydrodynamic -type description implies holographic implementations of dynamics through velocity fields at non-differentiable scale resolution, via fractal soliton, fractal soliton-kink, and fractal minimal vortex. The fractal Schrodinger-type description thus implies holographic implementation of complex system dynamics though in-phase coherences of fractal state fields via Airy fractal functions. In this last description, various operational procedures can become functional. We can mention the fractal cubes with fractal SL(2R) group invariance through in-phase coherence of the structural unit dynamics of any complex fluid, fractal $\mathrm{SL}(2 \mathrm{R})$ groups through dynamic synchronization among the complex system structural units, fractal Riemann manifolds induced by fractal cubics and embedded with a Poincaré metric through apolar transport of cubes, and harmonic mapping from the usual space to the hyperbolic one. These procedures become operational so that several possible scenarios towards chaos (fractal periodic doubling scenario), but without fully transitioning into chaos, (non-manifest chaos) can be obtained.

In this work, we will analyze from a multifractal perspective the nonlinear dynamics of complex systems, generalizing the results from [4]. In such context, exploring a hidden symmetry under the form of synchronization groups of complex system entities leads to the generation of a Riemann manifold with a hyperbolic type metric via parallel transport of direction. Then, accessing complex systems' nonstationary dynamics is performed thorough harmonic mapping from the usual space to the hyperbolic one.

\section{Mathematical Model}

\subsection{Motion Equation}

In the following, any complex system can be assimilated with a multifractal object. Then, since in the framework of scale relativity theory [6-9], the dynamics of complex system entities are described through continuous and non-differentiable curves (multifractal curves), the motion equation (with geodesics equation status) becomes (for detail see [6-9]):

$$
\frac{\hat{d} \hat{V}^{i}}{d t}=\partial_{t} \hat{V}^{i}+\hat{V}^{l} \partial_{l} \hat{V}^{i}+\frac{1}{4}(d t)^{\left[\frac{2}{f(\alpha)}\right]-1} D^{l k} \partial_{l} \partial_{k} \hat{V}^{i}=0,
$$

where

$$
\begin{gathered}
\hat{V}^{l}=V_{D}^{l}-V_{F}^{l} \\
D^{l k}=d^{l k}-i \hat{d}^{l k} \\
d^{l k}=\lambda_{+}^{l} \lambda_{+}^{k}-\lambda_{-}^{l} \lambda_{-}^{k} \\
\hat{d}^{l k}=\lambda_{+}^{l} \lambda_{+}^{k}+\lambda_{-}^{l} \lambda_{-}^{k} \\
\partial_{t}=\frac{\partial}{\partial t^{\prime}}, \partial_{l}=\frac{\partial}{\partial x^{l}}, \partial_{l} \partial_{k}=\frac{\partial}{\partial x^{l}} \frac{\partial}{\partial x^{k}}, i=\sqrt{-1}, l, k=1,2,3 .
\end{gathered}
$$


In relation (1), the meanings of the variables and parameters are as follows:

- $x^{l}$ is the multifractal spatial coordinate;

- $t$ is the non-multifractal time having the role of an affine parameter of the motion curves;

- $\quad \hat{V}^{l}$ is the multifractal complex velocity;

- $\quad V_{D}^{l}$ is the differentiable velocity independent of the scale resolution;

- $\quad V_{F}^{l}$ is the non-differentiable velocity dependent on the scale resolution;

- $\quad d t$ is the scale resolution;

- $f(\alpha)$ is the singularity spectrum of order $\alpha$;

- $\quad \alpha$ is the singularity index and is a function of fractal dimension $D_{f}$;

- $D^{l k}$ is the constant tensor associated with the differentiable-non-differentiable transition;

- $\quad \lambda_{+}^{l}\left(\lambda_{+}^{k}\right)$ is the constant vector associated with the backward differentiable-nondifferentiable dynamic processes;

- $\lambda_{-}^{l}\left(\lambda_{-}^{k}\right)$ is the constant vector associated with the forward differentiablenon-differentiable dynamic processes.

The relation (1) shows that in the most general case of complex system structural unit dynamics, regardless of the fractalization type, the multifractal inertial, $\partial_{t} \hat{V}^{i}$, the multifractal convective, $\hat{V}^{l} \partial_{l} \hat{V}^{i}$, and the multifractal dissipative effects, $\frac{1}{4}(d t)^{\left[\frac{2}{f(\alpha)}\right]-1} D^{l k} \partial_{l} \partial_{k} \hat{V}^{i}$, are achieving balance at any point of the movement curve.

By using the singularity spectrum, the following patterns in the complex system dynamics can be distinguished: monofractal patterns that imply dynamics in homogenous complex systems characterized though a single fractal dimension and having the same scaling properties in any time interval; multifractal patterns that include dynamics in inhomogeneous and anisotropic complex systems characterized simultaneously by a wide variety of fractal dimensions. Thus, $f(\alpha)$ allows the identification of the universality classes in the dynamics of any complex system even when the strange attractors associated with these dynamics have different aspects. For details on the singularity spectrum and its implication for the dynamics of complex systems, see [10-12].

\subsection{Schrodinger and Madelung Scenarios in the Description of Complex System Dynamics}

For a large temporal scale resolution with respect to the inverse of the highest Lyapunov exponent [7-9], the class of deterministic trajectories of any complex system entity can be substituted by the class of virtual trajectories. Then, the concept of definite trajectories is replaced by the one of density of probability. The multifractality is then expressed by means of multi-stochasticity and becomes functional when describing the dynamic of any complex system in the form of multifractal fluid dynamics (for details see [5-9]).

Many modes of multifractalization through stochasticization processes can be defined. Among the most utilized processes, the Markovian and non-Markovian stochastic processes are found [10-12]. In the following description of complex system dynamics, only multifractalizations by means of Markovian stochastic processes will be discussed, i.e., those specified by constraints [10-12]:

$$
\lambda_{+}^{i} \lambda_{+}^{l}=\lambda_{-}^{i} \lambda_{-}^{l}=2 \lambda \delta^{i l},
$$

where $\lambda$ is a constant associated with the differentiable-non-differentiable transitions and $\delta^{i l}$ is the Kronecker pseudo-tensor. Based on (3), the motion Equation (1) become (for details on the mathematical procedure see [7-9]):

$$
\frac{\hat{d} \hat{V}^{i}}{d t}=\partial_{t} \hat{V}^{i}+\hat{V}^{l} \partial_{l} \hat{V}^{i}-i \lambda(d t)^{\left[\frac{2}{f(\alpha)}\right]-1} \partial_{l} \partial^{l} \hat{V}^{i}=0 .
$$


The relation (4) shows that for the case of complex system structural unit dynamics, only for multifractalization by means of Markovian stochastic processes (for the case of Brownian or Levy type motions) in any point of the motion curves, the local multifractal complex acceleration, $\partial_{t} \hat{V}^{i}$, the multifractal complex convection, $\hat{V}^{l} \partial_{l} \hat{V}^{i}$, and the multifractal complex dissipation $i \lambda(d t)^{\left[\frac{2}{f(\alpha)}\right]-1} \partial_{l} \partial^{l} \hat{V}^{i}$ are in equilibrium.

In the following, let it be allowed that the motions of the entities belonging to any complex system are irrotational. Then, the multifractal complex velocity fields from (2) become:

$$
\hat{V}^{i}=-2 i \lambda(d t)^{\left[\frac{2}{f(\alpha)}\right]-1} \partial^{i} \ln \Psi,
$$

where

$$
\chi=-2 i \lambda(d t)^{\left[\frac{2}{f(\alpha)}\right]-1} \ln \Psi
$$

is the multifractal complex scalar potential of the complex velocity fields from (5) and $\Psi$ is the function of states (on the significance of $\Psi$, see [5-10]). In these conditions, substituting (5) in (4) and using the mathematical procedures from [6-9], the motion Equation (4) takes the form of the multifractal Schrödinger equation:

$$
\lambda^{2}(d t)^{\left[\frac{4}{f(\alpha)}\right]-2} \partial^{l} \partial_{l} \Psi+i \lambda(d t)^{\left[\frac{2}{f(\alpha)}\right]-1} \partial_{t} \Psi=0 .
$$

Therefore, for the complex velocity fields (5), the dynamics of any complex system entity are described through Schrödinger type "regimes" at various scale resolutions (Schrödinger's multifractal description). Equation (7) defines the Schrödinger scenario on the holographic implementation of complex system dynamics.

Moreover, if $\Psi$ is chosen in the form (Madelung's type choice):

$$
\Psi=\sqrt{\rho} e^{i s},
$$

where $\sqrt{\rho}$ is the amplitude and $s$ is the phase, then the multifractal complex velocity fields (5) take the explicit form:

$$
\hat{V}^{i}=2 \lambda(d t)^{\left[\frac{2}{f(\alpha)}\right]-1} \partial^{i} s-i \lambda(d t)^{\left[\frac{2}{f(\alpha)}\right]-1} \partial^{i} \ln \rho,
$$

which implies the real multifractal velocity fields:

$$
\begin{gathered}
V_{D}^{i}=2 \lambda(d t)^{\left[\frac{2}{f(\alpha)}\right]-1} \partial^{i} s \\
V_{F}^{i}=\lambda(d t)^{\left[\frac{2}{f(\alpha)}\right]-1} \partial^{i} \ln \rho .
\end{gathered}
$$
field.

In (10), $V_{D}^{i}$ is the differential velocity field, while in (11), $V_{F}^{i}$ is the multifractal velocity

By (9)-(11) and using the mathematical procedure from [6-10], the motion Equation (4) reduces to the multifractal Madelung equations:

$$
\begin{gathered}
\partial_{t} V_{D}^{i}+V_{D}^{l} \partial_{l} V_{D}^{i}=-\partial^{i} Q \\
\partial_{t} \rho+\partial_{l}\left(\rho V_{D}^{l}\right)=0,
\end{gathered}
$$

with $Q$ the multifractal specific potential:

$$
Q=-2 \lambda^{2}(d t)^{\left[\frac{4}{f(\alpha)}\right]-2} \frac{\partial^{l} \partial_{l} \sqrt{\rho}}{\sqrt{\rho}}=-V_{F}^{i} V_{F}^{i}-\frac{1}{2} \lambda(d t)^{\left[\frac{2}{f(\alpha)}\right]-1} \partial_{l} V_{F}^{l} .
$$


Equation (12) corresponds to the multifractal specific momentum conservation law, while Equation (13) corresponds to the multifractal states density conservation law. The multifractal specific potential (14) implies the multifractal specific force:

$$
F^{i}=-\partial^{i} Q=-2 \lambda^{2}(d t)^{\left[\frac{4}{f(\alpha)}\right]-2} \partial^{i} \frac{\partial^{l} \partial_{l} \sqrt{\rho}}{\sqrt{\rho}},
$$

which is a measure of the multifractality of the motion curves.

Therefore, for the multifractal complex velocity fields (9), the dynamics of any complex system are described through Madelung-type "regimes" at various scale resolutions (Madelung's multifractal description). Equations (12)-(14) define the Madelung scenario on the holographic implementation for complex system dynamics. In this context, any complex system entity is in a permanent interaction with a multifractal medium through the multifractal specific force (15). All complex systems can be identified with a multifractal fluid, the dynamics of which are described by the multifractal Madelung equations (see (12)-(14)). The velocity field $V_{F}^{i}$ does not represent the contemporary dynamics. Since $V_{F}^{i}$ is missing from (13), this velocity field contributes to the transfer of the multifractal specific momentum and to the multifractal energy focus. Any analysis of $Q$ should consider the "self" nature of the specific momentum transfer of multifractal type. Then, the conservation of the multifractal energy and the multifractal momentum ensure the reversibility and the existence of the multifractal eigenstates.

If the multifractal tensor is considered:

$$
\hat{\tau}^{i l}=2 \lambda^{2}(d t)^{\left[\frac{4}{f(\alpha)}\right]-2} \rho \partial^{i} \partial^{l} \ln \rho,
$$

the equation defining the multifractal forces that derive from the multifractal specific potential $Q$ can be written in the form of a multifractal equilibrium equation:

$$
\rho \partial^{i} Q=\partial_{l} \hat{\tau}^{i l}
$$

Since $\hat{\tau}^{i l}$ can be also written in the form:

$$
\hat{\tau}^{i l}=\eta\left(\partial_{l} V_{F}^{i}+\partial_{i} V_{F}^{l}\right)
$$

with

$$
\eta=\lambda(d t)^{\left[\frac{2}{f(\alpha)}\right]-1} \rho
$$

a multifractal linear constitutive equation for a multifractal "viscous fluid" can be highlighted. In such a context, the coefficient $\eta$ can be interpreted as a multifractal dynamic viscosity coefficient of the multifractal fluid.

\subsection{Synchronization Modes in Complex System Dynamics through a "Hidden" Symmetry}

The existence of multifractal specific force (15) and the multifractal viscosity tensor (16) will be considered as the "trigger" of the complex system processes that lead both to instabilities and to self-structuring. If the multifractal specific potential is constant, through (15) for the one-dimensional case, the following condition is satisfied:

$$
\frac{\partial^{2} \sqrt{\rho}}{\partial x^{2}}+k_{0}^{2} \sqrt{\rho}=0,
$$

with

$$
k_{0}^{2}=\frac{E}{2 \lambda^{2}(d t)^{\left[\frac{4}{f(\alpha)}\right]-2}} .
$$


In the above relation, $E$ is the multifractal energy of the complex system's entity and $m_{0}$ is the rest mass. The solution of (20) can be written in the form

$$
\sqrt{\rho}=z e^{i\left(k_{0} x+\theta\right)}+\bar{z} e^{-i\left(k_{0} x+\theta\right)},
$$

where $z$ is a complex amplitude, $\bar{z}$ is its complex conjugate, $\theta$ is a specific phase and $x$ is the multifractal spatial coordinate. In such a context, $z$ and $\theta$ "scan" each entity of the complex system, which has as a general characteristic Equation (20), and thus the same $k_{0}$.

Equation (20) has a multifractal hidden symmetry by means of a homographic group. Indeed, the ratio $\varepsilon$ of two independent linear solutions of Equation (20) is a solution of multifractal Schwartz's differential equation (for the classical case, see [6-9]):

$$
\begin{gathered}
\{\varepsilon, x\}=\left(\frac{\varepsilon^{\prime \prime}}{\varepsilon^{\prime}}\right)^{\prime}-\frac{1}{2}\left(\frac{\varepsilon^{\prime \prime}}{\varepsilon^{\prime}}\right)^{2}=2 k_{0}^{2} \\
\varepsilon^{\prime}=\frac{d \varepsilon}{d x}, \varepsilon^{\prime \prime}=\frac{d^{2} \varepsilon}{d x^{2}} .
\end{gathered}
$$

The left part of (22) is invariant with respect to the multifractal homographic transformation

$$
\varepsilon \leftrightarrow \varepsilon^{\prime}=\frac{a \varepsilon+b}{c \varepsilon+d}
$$

with $a, b, c, d$ multifractal real parameters. The relation (24) corresponding to all possible values of these parameters defines the multifractal group SL(2R) (for the classical case, see $[13,14])$.

Thus, all of the complex system entities having the same $k_{0}$ are in biunivocal correspondence with the transformation of the multifractal group SL(2R). This allows the construction of a personal parameter $\varepsilon$ for each individual complex system entity. Indeed, as a guide, it is chosen in the general form of solution of (22), which is written as

$$
\varepsilon^{\prime}=l+m \tan \left(k_{0} x+\theta\right)
$$

Thus, through $l, m$, and $\theta$, it is possible to characterize any complex systems entity. In such conjecture, identifying the phase from (25) with the one from (21), the personal parameter becomes:

$$
\varepsilon(x)=\frac{z+\bar{z} \varepsilon}{1+z}, z=l+i m, \bar{z}=l-i m, \varepsilon \equiv e^{2 i\left(k_{0} x+\theta\right)} .
$$

The fact that (25) is also a solution of (22) implies, by explicitly solving (24), that the multifractal group $\mathrm{SL}(2 \mathrm{R})$ :

$$
z^{\prime}=\frac{a z+b}{c z+d}, \bar{z}^{\prime}=\frac{a \bar{z}+b}{c \bar{z}+d^{\prime}}, \varepsilon^{\prime}=\frac{c \bar{z}+d}{c z+d} \varepsilon .
$$

Therefore, the multifractal group (27) works as a synchronization mode among various entities of any complex system process to which the amplitudes and the phases are also connected. More precisely, through (27) the phase of $\varepsilon$ is only moved with a quantity depending on the amplitude of the complex system at the transition among various complex system entities. Moreover, the amplitude of the movement is also affected from a multifractal homographic perspective. The usual synchronization modes manifested through delay of the amplitudes and phases of the complex system entities must describe here only a particular case.

\subsection{Riemann's Manifold Generated through Synchronization Processes}

According to the mathematical procedures from [6-9,15-17], the space of multifractal group (27) can be structured by means of $(z, \bar{z}, \varepsilon)$ parameters, as a multifractal Riemann's 
manifold. Indeed, the structure of multifractal group (27) is typical of an $S L(2 R)$ one, which is taken in the standard form

$$
\left[A_{1}, A_{2}\right]=A_{1},\left[A_{2}, A_{3}\right]=A_{3},\left[A_{3}, A_{1}\right]=-2 A_{2}
$$

where $A_{k}, k=1,2,3$ are the multifractal infinitesimal generators of the group. Since the multifractal group is simple transitive, these multifractal generators can be found as components of the multifractal Cartan coframe (for the classical case, see Cartan [15]) from the relation.

$$
d(f)=\sum \frac{\partial f}{\partial x^{k}} d x^{k}=\left\{\omega^{1}\left[z^{2} \frac{\partial}{\partial z}+\bar{z}^{2} \frac{\partial}{\partial \bar{z}}+(z-\bar{z}) \varepsilon \frac{\partial}{\partial \varepsilon}\right]+2 \omega^{2}\left(z \frac{\partial}{\partial z}+\bar{z} \frac{\partial}{\partial \bar{z}}\right)+\omega^{3}\left(\frac{\partial}{\partial z}+\frac{\partial}{\partial \bar{z}}\right)\right\}(f)
$$

where $\omega^{k}$ are the components of the multifractal Cartan coframe which can be found from the system:

$$
d z=\omega^{1} z^{2}+2 \omega^{2} z+\omega^{3}, d \bar{z}=\omega^{1} \bar{z}^{2}+2 \omega^{2} \bar{z}+\omega^{3}, d \varepsilon=\omega^{1} \mathcal{\varepsilon}(z-\bar{z})
$$

Thus, both the multifractal infinitesimal generators and the multifractal coframe are obtained by identifying the right-hand side of (29) with the standard dot product of multifractal algebra $S L(2 R)$

$$
\omega^{1} A_{3}+\omega^{3} A_{1}-2 \omega^{2} A_{2}
$$

so that

$$
A_{1}=\frac{\partial}{\partial z}+\frac{\partial}{\partial \bar{z}}, \quad A_{2}=z \frac{\partial}{\partial z}+\bar{z} \frac{\partial}{\partial \bar{z}}, \quad A_{3}=z^{2} \frac{\partial}{\partial z}+\bar{z}^{2} \frac{\partial}{\partial \bar{z}}+(z-\bar{z}) \varepsilon \frac{\partial}{\partial \varepsilon}
$$

and

$$
\omega^{1}=\frac{d \varepsilon}{(z-\bar{z}) \varepsilon}, \quad 2 \omega^{2}=\frac{d z-d \bar{z}}{z-\bar{z}}-\frac{z+\bar{z}}{z-\bar{z}} \frac{d \varepsilon}{\varepsilon}, \quad \omega^{3}=\frac{z d \bar{z}-\bar{z} d z}{z-\bar{z}}+\frac{z \bar{z} d \varepsilon}{(z-\bar{z}) \varepsilon} .
$$

In real terms from (26), these last multifractal equations can be written as

$$
\begin{gathered}
A_{1}=\frac{\partial}{\partial l}, \quad A_{2}=l \frac{\partial}{\partial l}+m \frac{\partial}{\partial m}, \quad A_{3}=\left(l^{2}-m^{2}\right) \frac{\partial}{\partial l}+2 l m \frac{\partial}{\partial m}+2 m \frac{\partial}{\partial \theta} \\
\omega^{1}=\frac{d \theta}{2 m}, \quad \omega^{2}=\frac{d m}{m}-\frac{l}{m} d \theta, \omega^{2}=\frac{l^{2}+m^{2}}{2 m} d \theta+\frac{m d l-l d m}{m} .
\end{gathered}
$$

It should be mentioned that in [6-9], it does not work with the previous multifractal differential forms, but with the multifractal absolute invariant differentials:

$$
\omega^{1}=\frac{d z}{(z-\bar{z}) \varepsilon}, \quad \omega^{2}=-i\left(\frac{d \varepsilon}{\varepsilon}-\frac{d z+d \bar{z}}{z-\bar{z}}\right), \quad \omega^{3}=\frac{-\varepsilon d \bar{z}}{z-\bar{z}}
$$

or, in real terms, exhibiting a three-dimensional Lorentz structure of this multifractal space

$$
\Omega^{1}=\omega^{1}=d \theta+\frac{d l}{m}, \Omega^{2}=\cos \theta \frac{d l}{m}+\sin \theta \frac{d m}{m}, \Omega^{3}=-\sin \theta \frac{d l}{m}+\cos \theta \frac{d m}{m}
$$

The advantage of this representation is that it makes obvious the multifractal connection with the multifractal Poincare representation of the multifractal Lobachevsky plane. Indeed, the multifractal metric is:

$$
\frac{d s^{2}}{g}=\left(\omega^{2}\right)^{2}-4 \omega^{1} \omega^{2}=\left(\frac{d \varepsilon}{\varepsilon}-\frac{d z+d \bar{z}}{z-\bar{z}}\right)^{2}+4 \frac{d z d \bar{z}}{(z-\bar{z})^{2}}
$$

or in real terms

$$
-\frac{d s^{2}}{g}=-\left(\Omega^{1}\right)^{2}+\left(\Omega^{2}\right)^{2}+\left(\Omega^{3}\right)^{2}=-\left(d \theta+\frac{d l}{m}\right)^{2}+\frac{d l^{2}+d m^{2}}{m^{2}},
$$


where $g$ is a multifractal constant.

This multifractal metric reduces to that of Poincaré

$$
\frac{d s^{2}}{g}=-4 \frac{d z d \bar{z}}{(z-\bar{z})^{2}}=-4 \frac{d l^{2}+d m^{2}}{m^{2}} .
$$

in the case when $\omega^{2}=0$ or $\Omega^{1}=0$, which defines the variable $\theta$ as the "angle of parallelism" (in Levi-Civita sense) of the multifractal hyperbolic plane (the multifractal connection). The multifractal Riemann manifold can further be associated with particular coherence domains induced by the parallel transport of direction. In fact, if in modern terms $\frac{d l}{m}$ represents the multifractal connection form of the multifractal hyperbolic plane, the relations in (37) then represent a general multifractal Bäcklung transformation in that multifractal plane. For the classical case, see [16].

\subsection{Complex System Dynamics via Harmonic Mapping}

In the following, we will generate non-stationary dynamics in complex systems through harmonic map generation. Indeed, let us assume that the complex system dynamics are described by the variables $\left(Y^{j}\right)$, for which the following multifractal metric was discovered:

$$
h_{i j} d Y^{i} d Y^{j}
$$

in an ambient space of multifractal metric:

$$
\gamma_{\alpha \beta} d X^{\alpha} d X^{\beta}
$$

In this situation, the field equations of the complex system dynamics are derived from a variational principle, connected to the multifractal Lagrangian:

$$
L=\gamma^{\alpha \beta} h_{i j} \frac{d Y^{i} d Y^{j}}{\partial X^{\alpha} \partial X^{\beta}}
$$

In the current case, (40) is given by (39) with the constraint $\omega^{2}=0$, the field variables being $z$ and $\bar{z}$ or, equivalently, the real and imaginary part of $z$. Therefore, if the variational principle:

$$
\delta \int L \sqrt{\gamma} d^{3} x
$$

is accepted as a starting point, where $\gamma=\left|\gamma_{\alpha \beta}\right|$, the main purpose of the complex system dynamics research would be to produce multifractal metrics of the multifractal Lobachevski plane (or related to it). In such a context, the multifractal Euler equations corresponding to the variational principle (43) are:

$$
\begin{aligned}
& (z-\bar{z}) \nabla(\nabla \mathrm{z})=2(\nabla \mathrm{z})^{2} \\
& (z-\bar{z}) \nabla(\nabla \bar{z})=2(\nabla \bar{z})^{2}
\end{aligned}
$$

which allows the solution:

$$
h=\frac{\cosh \left(\frac{\Phi}{2}\right)-\sinh \left(\frac{\Phi}{2}\right) e^{-i \alpha}}{\cosh \left(\frac{\Phi}{2}\right)+\sinh \left(\frac{\Phi}{2}\right) e^{-i \alpha}}, \alpha \in \mathbb{R},
$$

with $\alpha$ real and arbitrary, as long as $\left(\frac{\Phi}{2}\right)$ is the solution of a Laplace-type equation for the free space, such that $\nabla^{2}\left(\frac{\Phi}{2}\right)=0$. For a choice of the form $\alpha=2 \Omega t$, in which case a temporal dependency was introduced in the complex system dynamics, (45) becomes: 


$$
h=\frac{i\left[e^{2 \Phi} \sin (2 \Omega t)-\sin (2 \Omega t)-2 i e^{\Phi}\right]}{e^{2 \Phi}[\cos (2 \Omega t)+1]-\cos (2 \Omega t)+1} .
$$

In Figures 1-3, multiple nonlinear behaviors of complex dynamics at scale resolutions in dimensionless coordinates are presented via Python simulations: (i) nonlinear behaviors at a global scale resolution (Figure 1a,b); (ii) nonlinear behaviors at a differentiable scale resolution (Figure 2a,b); (iii) nonlinear behaviors at a non-differentiable scale resolution (Figure 3a,b). Let it be noted that, whatever the scale resolution, complex system dynamics prove themselves to be reducible to self-structuring patterns. The structures are present in pairs of two large patterns that are intercommunicated in an intermittent way. In the 0 -20 range for $\Omega$ and $t$, the resulting structures are communicating with each other via a channel created along the symmetry axis for $t \sim 10$. This channel is also seen for different $(\Omega ; t)$ coordinates, which is interpreted as an intermittency in the structure bonding. Based on the properties of the studied system, there are some associations with real physical phenomena that can be made. The self-structuring process is a well-known aspect of lowtemperature plasmas $[18,19]$. In recent years there have been some reports on structuring of the laser-produced plasmas [19-21], with impacts in pulsed laser deposition technology. The data presented here can be correlated with the plasma structuring (into a fast structure and a slow structure, also named Coulomb and thermal structure, respectively, after the dominant ejection mechanism) during expansion based on the ablation mechanism and ionization state [21-23]. In recent years, a change in the understanding of this structure has been reported, and a separation based on the ionization state was more plausible for the energetic structuring of the plasma [21,24-26]. In a series of papers [26-30], it was shown that each structure can be correlated with certain properties of the target. For this reason, the use of a multifractal model would be suitable for understanding plasma structuring and exploring the relation between the structure, which, as of now, is outside the reach of any of the tools used [31,32]. The model shows that for the structuring process, a communication channel is formed that will automatically appear. If the same rational treatment is applied to the study of plasma structuring, we identify that these channels are the double layer forming at the interface between the two structures. Comments on the effect of the double layer separating the two-plasma structure were made in [28-32], and it was shown that it plays an important role in controlling the kinetics of laser-produced plasmas. Our model highlights an important aspect of the plasma double layers: they are 3-dimensional objects with different properties seen at different investigation scales that transcend the planar expansion. This is seen from Figures 1-3, where we see that the channel is present for different $(\Omega ; t)$ coordinates. The transcendence of the plasma double layer over several resolution scales is understandable, as the average value is of a few tens of Debye lengths [32], which is the core resolution scale in plasma physics. The presence of a transient double layer driving the dynamics of a laser-produced plasma is relatively novel and has been investigated through other modeling approaches and experimental investigations.

Let it be noted that the mathematical formalism of the multifractal theory of motion naturally implies various operational procedures (invariance groups, harmonic mappings, group isomorphisms, embedding manifolds, etc.) with quite a number of applications in complex systems and plasma physics dynamics [32]. Plotting $h$, once again in dimensionless parameters, also highlights certain temporal self-similar properties, with the multifractal structures being contained into similar multifractal structures at much higher scales (Figure $4 \mathrm{a}-\mathrm{c}$ ). Let us also note that the structure's communication channel has an exponential decrease in the $(\Omega ; t)$ plane, which reflects the dissipation processes [32] occurring during laser-produced plasma expansion. When they expand, laser-produced plasmas lose particles and energy through collisional/radiative processes. This will be reflected in the weakening of the plasma double layer and limiting of the reach to a small plasma volume in the proximity of the double layer. The model manages to express the dissipation of the plasma through the reduction of the channel amplitude on the $\Omega$ axis as the time variable is 
increased. This result represents an important step forward in understating the dynamics at the front of the plume. Most of plasma diagnostics and even modeling are concerned with late-time interactions mostly occurring in the core of the plasma. Our model manages to capture, albeit in a multifractal picture, dissipation processes and possible recombination occurring at the front of the plume. Expanding the reach of our results, we could find future implementation for pulsed-laser deposition, where the front of a subsequent plasma always interacts with the already-deposited film.

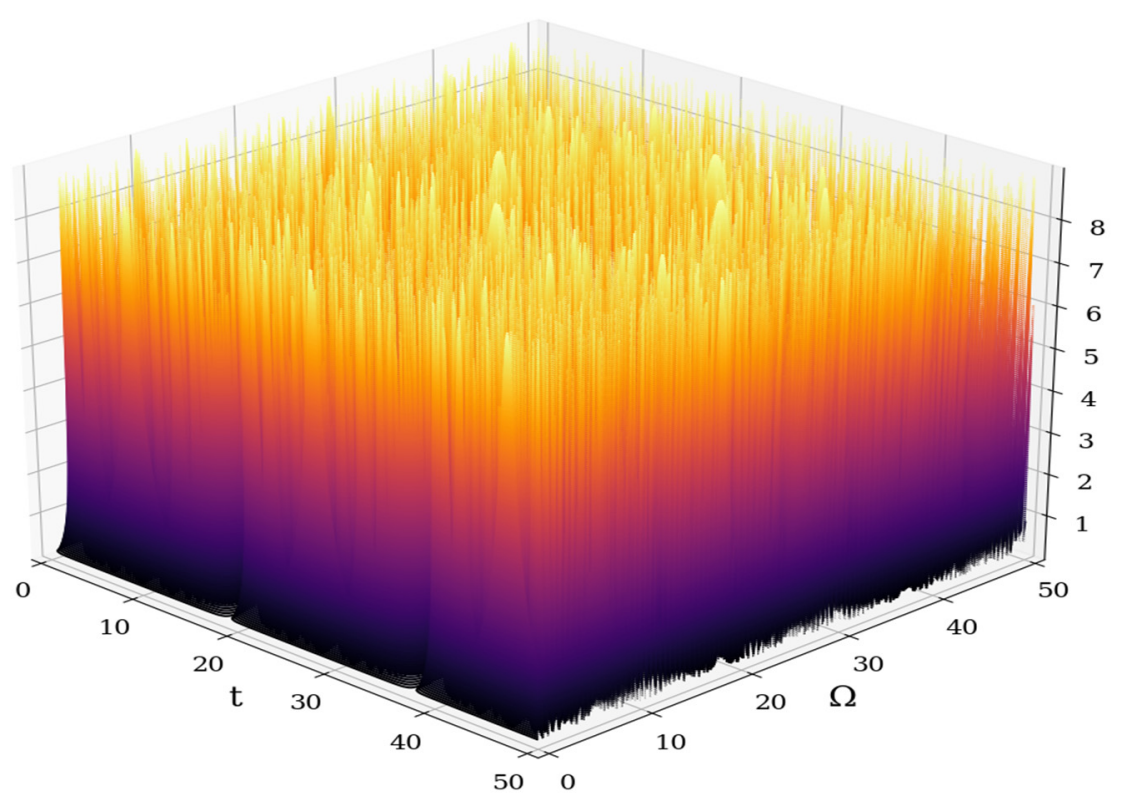

(a)

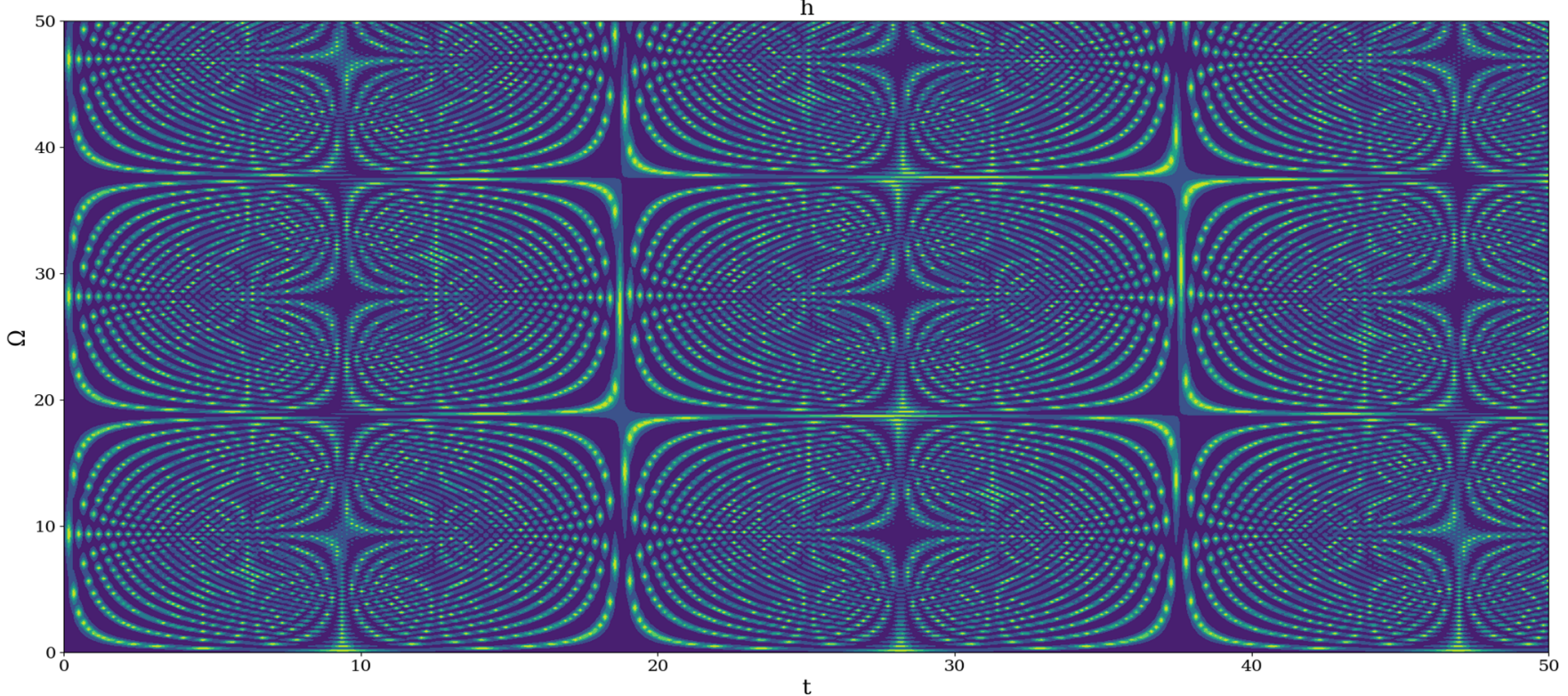

(b)

Figure 1. (a): 3D dynamics at global scale resolution of $h(\Omega, t)$ with $\Phi=2.35$. (b): 2D dynamics at global scale resolution of $h(\Omega, t)$ with $\Phi=2.35$. 


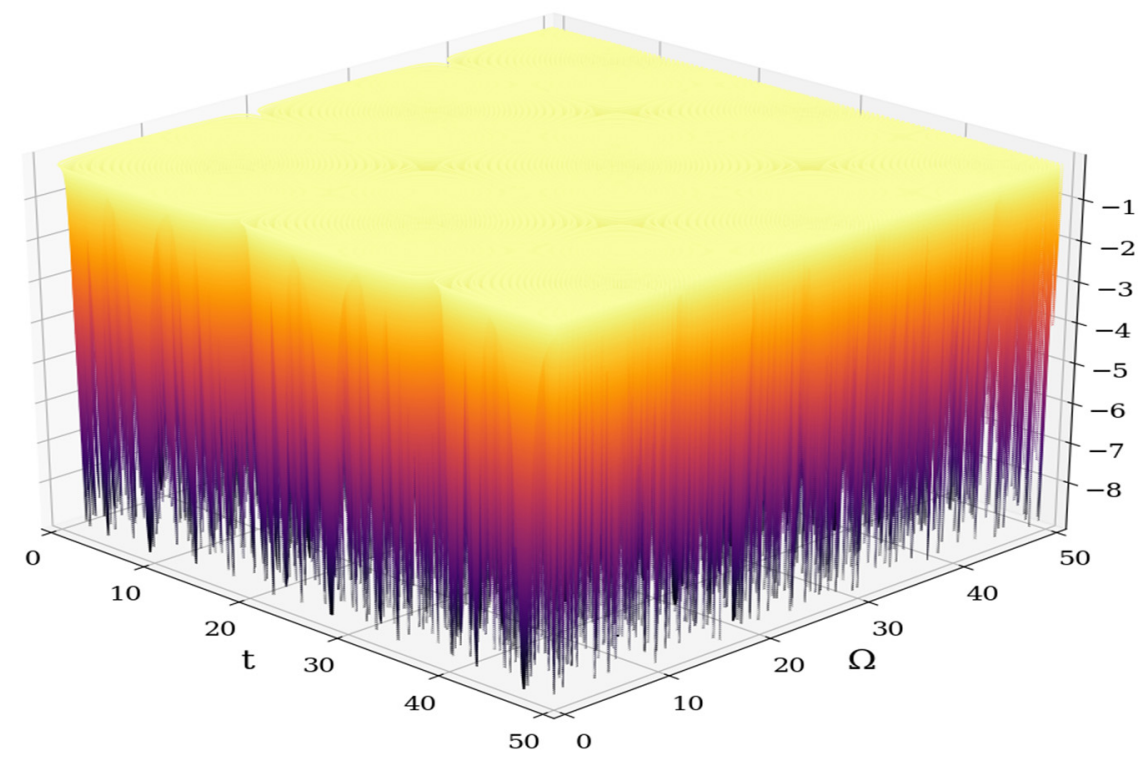

(a)

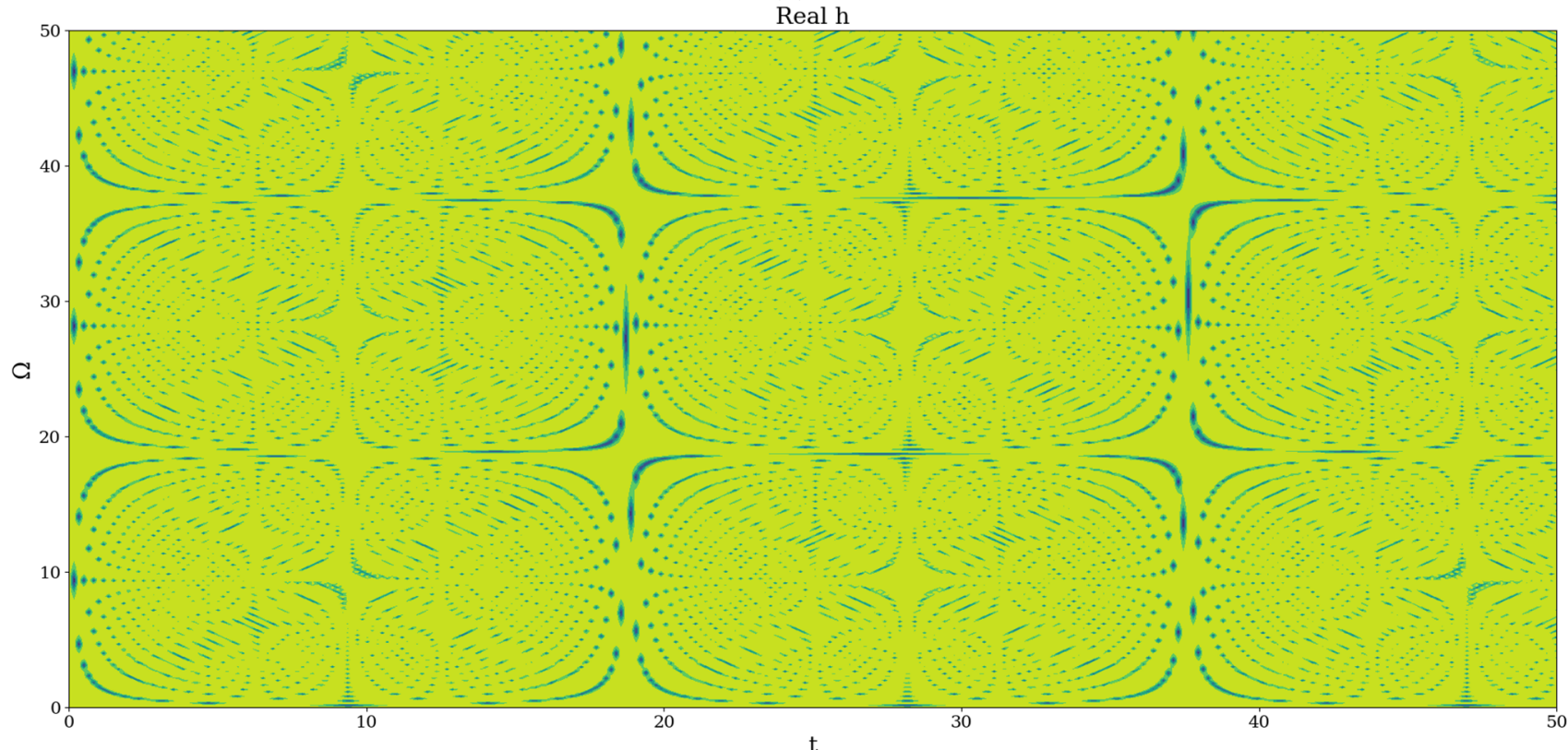

(b)

Figure 2. (a): 3D dynamics at differentiable scale resolution of $\operatorname{Re}[h(\Omega, t)]$ with $\Phi=2.35$.(b): $2 \mathrm{D}$ dynamics at differentiable scale resolution of $\operatorname{Re}[h(\Omega, t)]$ with $\Phi=2.35$. 


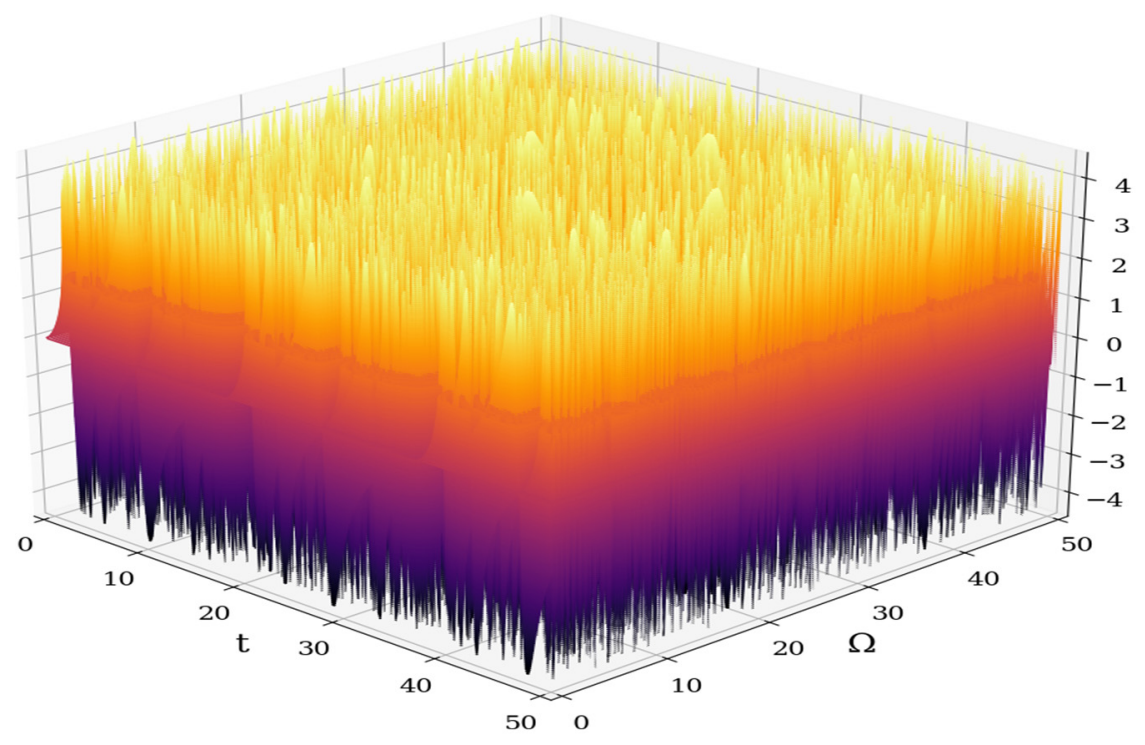

(a)

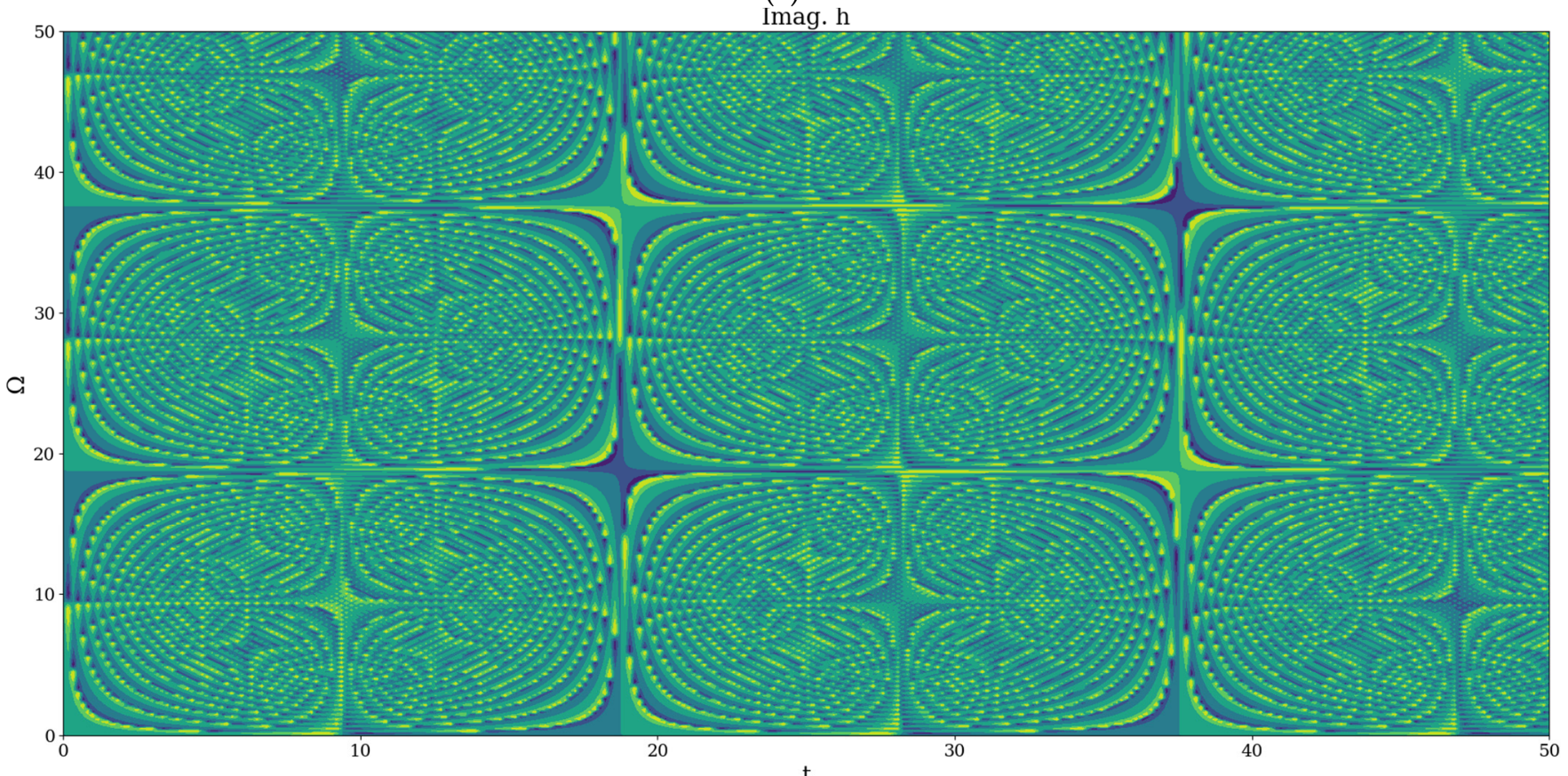

(b)

Figure 3. (a): $3 \mathrm{D}$ dynamics at non-differentiable scale resolution of $\operatorname{Im}[h(\Omega, t)]$ with $\Phi=2.35$. (b): $2 \mathrm{D}$ dynamics at non-differentiable scale resolution of $\operatorname{Im}[h(\Omega, t)]$ with $\Phi=2.35$.

The results presented in Figure 4a-c also specify that, through self-structuring of the complex system entities, channel-type patterns can also be observed. 


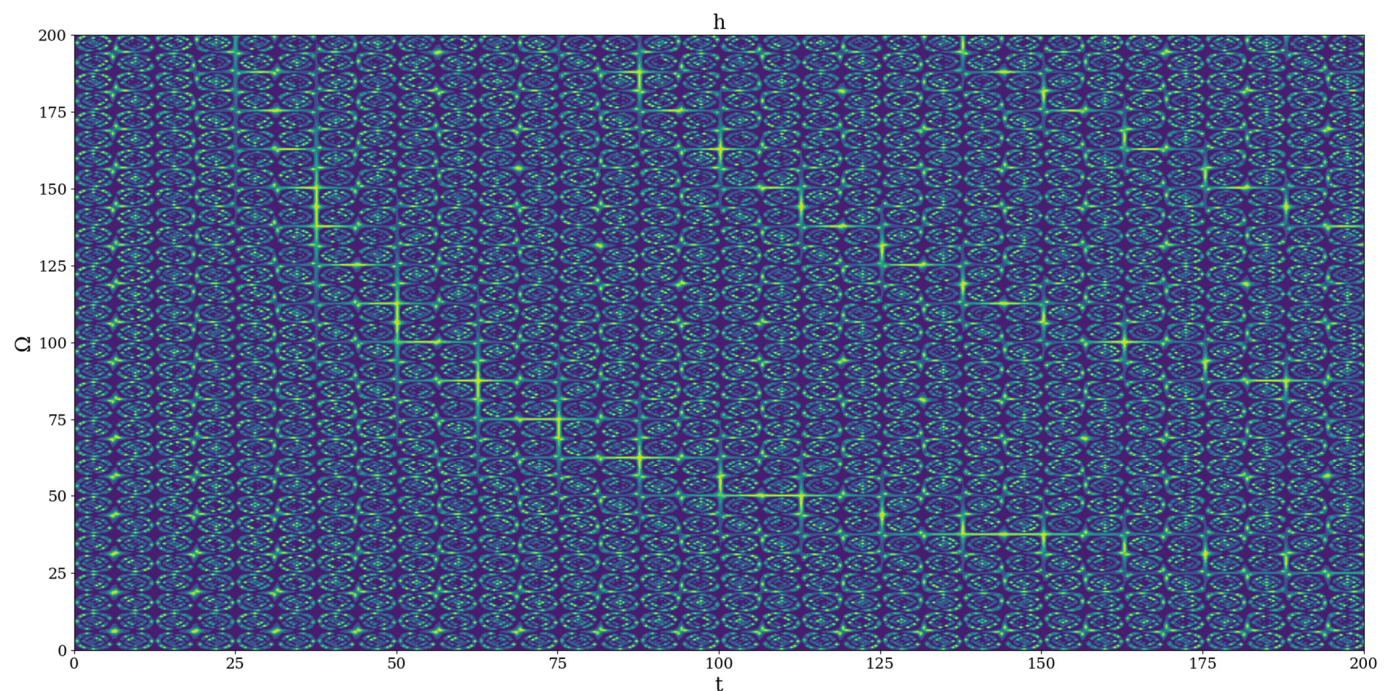

(a)

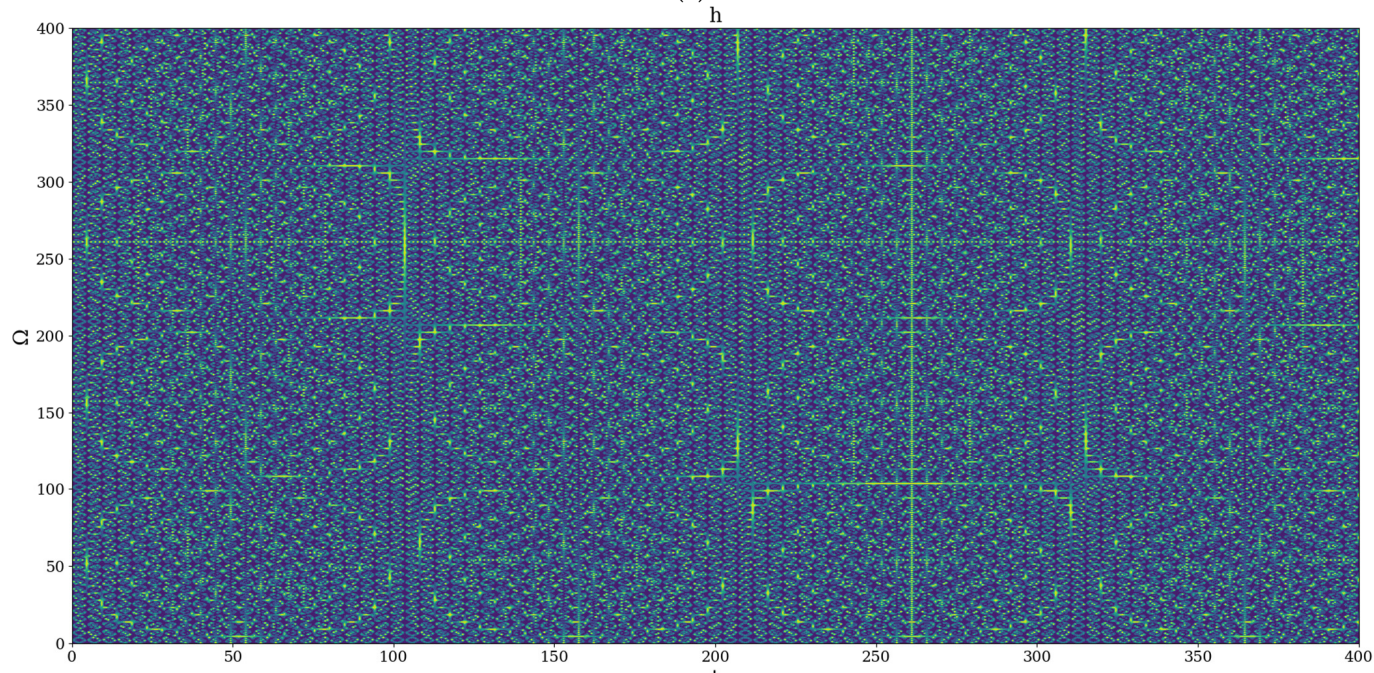

(b)

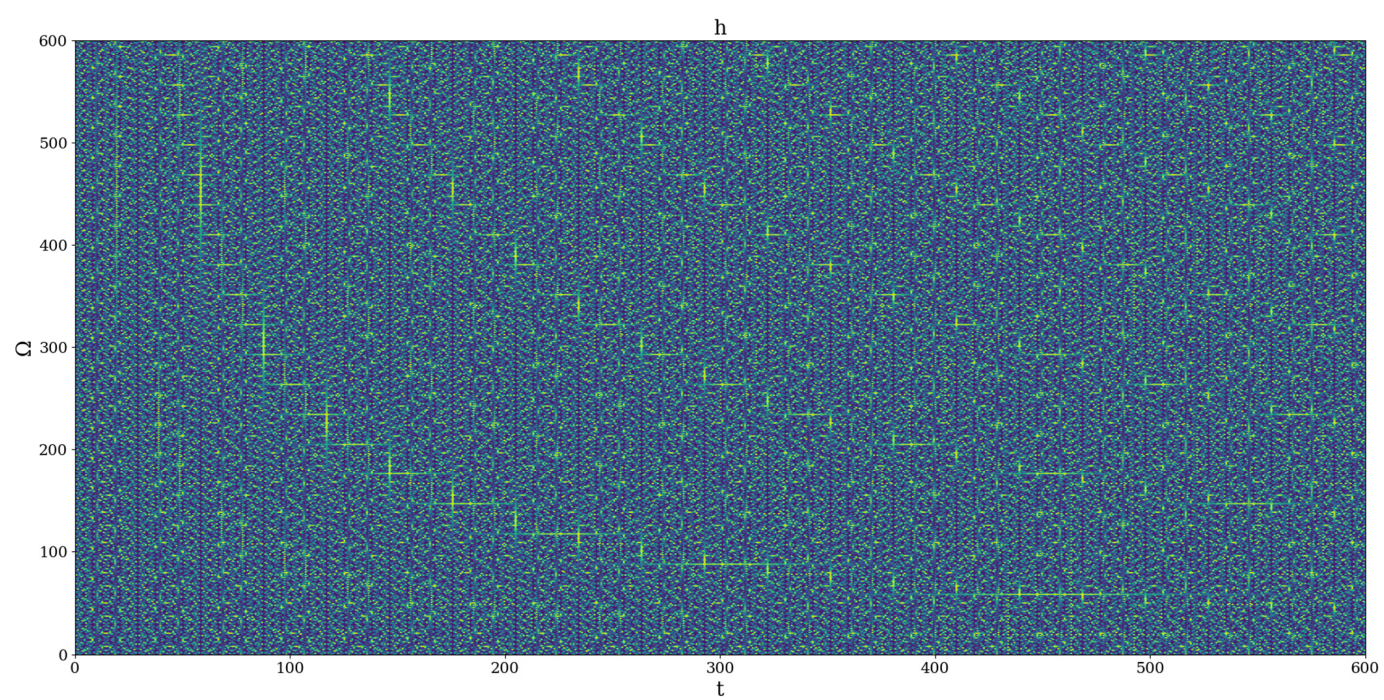

(c)

Figure 4. (a): $2 \mathrm{D}$ dynamics at global scale resolution of $h(\Omega=0-200, t=0-200) ; h=5$. (b): 2D dynamics at global scale resolution of $h(\Omega=0-400, t=0-400) ; h=5$. (c): 2D dynamics at global scale resolution of $h(\Omega=0-600, t=0-600)$; $h=5$. 


\section{Conclusions}

By considering that any complex system dynamics can be assimilated with a mathematical object of multifractal type, various non-linear behaviors in the framework of the scale relativity theory of motion are developed. In such a context, Schrödinger's and Madelung's holographic implementation scenarios for any complex system dynamics become operational through the multifractal motion curves. Exploring at various scale resolutions a hidden symmetry of stationary dynamics in the Madelung description, synchronization modes are seen forming through the SL (2R) group between the complex system entities. In the synchronization process, the amplitudes and phase of the motions of any complex system entity are shown to be connected, while the amplitude attributed to each motion can be tailored from a multifractal homographic perspective. The usual synchronization modes were proved to be manifested through the delay of the amplitude and phases of the complex system entities, and are here a particular case. The space induced by means of SL(2R) group parameters was structured at various scale resolutions as a Riemann manifold (multifractal Riemann manifold). The generators of a special Cartan coframe and their associated metrics were found. When a parallel transport of direction in the Levi-Civita sense became functional, the metric was reduced to that of Poincare, with the angle of parallelism of the hyperbolic plane defining the connections. Riemann manifolds were associated with coherence domains, with the coherence on each domain being induced by parallel transport of direction. Access to non-stationary dynamics at various scale resolutions became possible via harmonic mapping from the usual space to the hyperbolic one. Then, self-structuring of cellular and channel types were produced. The results are discussed with possible interpretations for the dynamics of laser-produced plasmas.

Author Contributions: Conceptualization, M.A. and S.A.I.; methodology, G.G. and L.T.; investigation, M.G., A.M.R. and S.A.I.; resources, G.G. and M.G.; writing—original draft preparation, S.A.I., and M.A.; writing — review and editing, M.A. and S.A.I. All authors have read and agreed to the published version of the manuscript.

Funding: This research received no external funding.

Institutional Review Board Statement: Not applicable.

Informed Consent Statement: Not applicable.

Data Availability Statement: Data will be available on request.

Acknowledgments: We would like to acknowledge the anonymous reviewers for their insightful comments that helped improve the manuscript.

Conflicts of Interest: The authors declare no conflict of interest.

\section{References}

1. Djebali, R.; Mebarek-Oudina, F.; Rajashekhar, C. Similarity solution analysis of dynamic and thermal boundary layers: Further formulation along a vertical flat plate. Phys. Scr. 2021, 96, 085206. [CrossRef]

2. Hamrelaine, S.; Mebarek-Oudina, F.; Sari, M.R. Analysis of MHD Jeffery Hamel Flow with Suction/Injection by Homotopy Analysis Method. J. Adv. Res. Fluid Mech. Ther. Sci. 2020, 58, 173-186.

3. Alkasassbeh, M.; Omar, Z.; Mebarek-Oudina, F.; Raza, J.; Chamkha, A. Heat transfer study of convective fin with temperaturedependent internal heat generation by hybrid block method. Heat Transf. Asian Res. 2019, 48, 1225-1244. [CrossRef]

4. Saviuc, A.; Gîrțu, M.; Topliceanu, L.; Petrescu, T.-C.; Agop, M. “Holographic Implementations” in the Complex Fluid Dynamics through a Fractal Paradigm. Mathematics 2021, 9, 2273. [CrossRef]

5. Nottale, L. Scale Relativity and Fractal Space-Time: A New Approach to Unifying Relativity and Quantum Mechanics; Imperial College: London, UK, 2011.

6. Merches, I.; Agop, M. Differentiability and Fractality in Dynamics of Physical Systems; World Scientific: Hackensack, NJ, USA, 2016.

7. Agop, M.; Paun, V.P. On the new perspectives of fractal theory. In Fundaments and Applications; Romanian Academy Publishing House: Bucharest, Romania, 2017.

8. Mazilu, N.; Agop, M.; Merches, I. Scale Transitions as Foundations of Physics; World Scientific: Singapore, 2021. 
9. Maziulu, N.; Agop, M.; Merches, I. The mathematical principles of scale relativity theory. In The Concept of Interpretation; CRC Press, Taylor and Francis Group: Boca Raton, FL, USA, 2020.

10. Strogatz, S.H. Nonlinear Dynamics and Chaos, 2nd ed.; CRC Press: Boca Raton, FL, USA, 2015.

11. Cristescu, C.P. Nonlinear dynamics and chaos. In Theoretical Fundaments and Applications; Romanian Academy Publishing House: Bucharest, Romania, 2008.

12. Mandelbrot, B.B. Fractal and Chaos; Springer: Berlin/Heidelberg, Germany, 2004.

13. Isaacs, I.M. Finite Group Theory American Mathematical Society; Providence: Rhode Island, RI, USA, 2008.

14. Ramadevi, P.; Dubey, V. Group Theory for Physicists with Applications; Cambridge University Press: Cambridge, UK, 2019.

15. Cartan, I. Riemannian Geometry in an Orthogonal Frame; World Scientific: Singapore, 2001.

16. Flanders, H. Differential Forms with Applications to the Physical Sciences; Dover Publication, Inc.: New York, NY, USA, 2012.

17. Felsager, B. Geometry, Particle and Fields; Springer: New York, NY, USA, 1998.

18. Dimitriu, D.G.; Irimiciuc, S.A.; Popescu, S.; Agop, M.; Ionita, C.; Schrittwieser, R.W. On the interaction between two fireballs in low-temperature plasma. Phys. Plasmas 2015, 22, 113511. [CrossRef]

19. Volkov, N.A. Splitting of laser-induced neutral and plasma plumes: Hydrodynamic origin of bimodal distributions of vapor density and plasma emission intensity. J. Phys. D Appl. Phys. 2021, 54, 37LT01. [CrossRef]

20. Irimiciuc, S.A.; Hodoroaba, B.C.; Bulai, G.; Gurlui, S.; Craciun, V. Multiple structure formation and molecule dynamics in transient plasmas generated by laser ablation of graphite. Spectrochim. Acta-Part B At. Spectrosc. 2020, 165, 105774. [CrossRef]

21. Kumar, R. Self-structuring in Laser-Blow-Off Plasma Plume. Int. J. Sci. Eng. Res. 2012, 3, 1-9.

22. Irimiciuc, S.A.; Chertopalov, S.; Craciun, V.; Novotný, M.; Lancok, J. Investigation of laser-produced plasma multistructuring by floating probe measurements and optical emission spectroscopy. Plasma Process. Polym. 2020, 11, 2000136. [CrossRef]

23. Morozov, A.A.; Evtushenko, A.B.; Bulgakov, A.V. Gas-dynamic acceleration of laser-ablation plumes: Hyperthermal particle energies under thermal vaporization. Appl. Phys. Lett. 2015, 106, 054107. [CrossRef]

24. Baraldi, G.; Perea, A.; Afonso, C.N. Dynamics of ions produced by laser ablation of several metals at $193 \mathrm{~nm}$. J. Appl. Phys. 2011, 109, 043302. [CrossRef]

25. Leitz, K.H.; Redlingsshofer, B.; Reg, Y.; Otto, A.; Schmidt, M. Metal Ablation with Short and Ultrashort Laser Pulses. Phys. Procedia 2011, 12, 230-238. [CrossRef]

26. Anoop, K.K.; Polek, M.P.; Bruzzese, R.; Amoruso, S.; Harilal, S.S. Multidiagnostic analysis of ion dynamics in ultrafast laser ablation of metals over a large fluence range. J. Appl. Phys. 2015, 117, 083108. [CrossRef]

27. Irimiciuc, S.A.A.; Gurlui, S.; Nica, P.; Focsa, C.; Agop, M. A compact non-differential approach for modeling laser ablation plasma dynamics. J. Appl. Phys. 2017, 121, 083301. [CrossRef]

28. Williams, G.O.; O'Connor, G.M.; Mannion, P.T.; Glynn, T.J. Langmuir probe investigation of surface contamination effects on metals during femtosecond laser ablation. Appl. Surf. Sci. 2008, 254, 5921-5926. [CrossRef]

29. Skočić, M.; Dojić, D.; Bukvić, S. Formation of double-layer in the early stage of nanosecond laser ablation. J. Quant. Spectrosc. Radiat. Transf. 2019, 227, 57-62. [CrossRef]

30. Eliezer, S.; Nissim, N.; Martínez Val, J.M.; Mima, K.; Hora, H. Double layer acceleration by laser radiation. Laser Part. Beams 2014, 32, 211-216. [CrossRef]

31. Beilis, I. Modeling of the plasma produced by moderate energy laser beam interaction with metallic targets: Physics of the phenomena. Laser Part. Beams 2012, 30, 341-356. [CrossRef]

32. Kokai, F.; Takahashi, K.; Shimizu, K.; Yudasaka, M.; Iijima, S. Shadowgraphic and emission imaging spectroscopic studies of the laser ablation of graphite in an Ar gas atmosphere. Appl. Phys. A Mater. Sci. Process. 1999, 69, 223-227. [CrossRef] 